\title{
BMJ Open Cross-sectional study of prevalence and risk factors, and a cost-effectiveness evaluation of screening and preventive treatment strategies for latent tuberculosis among migrants in Singapore
}

Vanessa W Lim, ${ }^{1}$ Hwee Lin Wee, ${ }^{2}$ Phoebe Lee,,${ }^{2,3}$ Yijun Lin,,${ }^{2,3}$ Yi Roe Tan, ${ }^{1}$ Mei Xuan Tan, ${ }^{1}$ Lydia Wenxin Lin, ${ }^{2}$ Peiling Yap, ${ }^{1}$ Cynthia BE Chee, ${ }^{4}$ Timothy Barkham (ID) , ${ }^{5}$ Vernon Lee, ${ }^{2,3}$ Mark Chen (D) , ${ }^{1}$ Rick Twee-Hee Ong ${ }^{2}$

To cite: Lim VW, Wee HL, Lee P, et al. Cross-sectional study of prevalence and risk factors, and a cost-effectiveness evaluation of screening and preventive treatment strategies for latent tuberculosis among migrants in Singapore. BMJ Open 2021;11:e050629. doi:10.1136/ bmjopen-2021-050629

- Prepublication history and additional online supplemental material for this paper are available online. To view these files, please visit the journal online. To view these files, please visit the journal online (http://dx.doi.org/10.1136/ bmjopen-2021-050629).

Received 05 March 2021 Accepted 01 July 2021

Check for updates

(c) Author(s) (or their employer(s)) 2021. Re-use permitted under CC BY-NC. No commercial re-use. See rights and permissions. Published by BMJ.

For numbered affiliations see end of article.

Correspondence to

Dr Mark Chen;

mark_ic_chen@ncid.sg

\section{ABSTRACT}

Objectives WHO recommends that low burden countries consider systematic screening and treatment of latent tuberculosis infection (LTBI) in migrants from high incidence countries. We aimed to determine LTBI prevalence and risk factors and evaluate costeffectiveness of screening and treating LTBI in migrants to Singapore from a government payer perspective. Design Cross-sectional study and cost-effectiveness analysis.

Setting Migrants in Singapore.

Participants 3618 migrants who were between 20 and 50 years old, have not worked in Singapore previously and stayed in Singapore for less than a year were recruited.

Primary and secondary outcome measures Costs, quality-adjusted life-years (QALYs), threshold length of stay, incremental cost-effectiveness ratios (ICERs), cost per active TB case averted.

Results 0 3584 migrants surveyed, 20.4\% had positive interferon-gamma release assay (IGRA) results, with the highest positivity in Filipinos (33.2\%). Higher LTBI prevalence was significantly associated with age, marital status and past TB exposure. The cost-effectiveness model projected an ICER of S\$57116 per QALY and S\$12 422 per active TB case averted for screening and treating LTBI with 3 months once weekly isoniazid and rifapentine combination regimen treatment compared with no screening over a 50-year time horizon. ICER was most sensitive to the cohort's length of stay in Singapore, yearly disease progression rates from LTBI to active TB, followed by the cost of IGRA testing.

Conclusions For LTBI screening and treatment of migrants to be cost-effective, migrants from high burden countries would have to stay in Singapore for $\sim 50$ years. Risk-stratified approaches based on projected length of stay and country of origin and/or age group can be considered.
Strengths and limitations of this study

- We measured age-stratified latent tuberculosis infection prevalence in migrants from eight high burden Asian countries which are sources of immigration not just for Singapore but also in many higher-income countries.

- Model parameters includes age-specific rates for TB progression, mortality due to TB and background mortality.

- Considerable uncertainty and variability about control measures and onward transmission impact on reactivation of latent TB.

\section{INTRODUCTION}

WHO guidelines for the programmatic management of latent tuberculosis infection (LTBI) suggests that systematic screening of immigrants from high TB burden countries could be considered in low TB burden countries. ${ }^{1}$ Furthermore, results from costeffectiveness studies on LTBI screening from low TB burden host countries in Europe, Canada and USA have shown that it is costeffective but with a wide range of incremental cost-effectiveness ratios (ICERs) from being highly cost-effective in Europe E $^{23}$ and Canada ${ }^{4}$ to borderline cost-effective in USA. ${ }^{5}$

For the many medium TB burden countries that have large number of immigrants, even less evidence is readily available for decision making. Singapore is classified as a medium TB burden country, ${ }^{6}$ but migrants residing in Singapore accounted for $49 \%$ of all notified cases of active TB in 2017. ${ }^{7}$ A substantial number of these migrants are from high TB burden countries such as Philippines, 
India, Myanmar and Indonesia, and these countries are also major contributors of migration to other regions in East Asia and the Middle-East. ${ }^{8}$ As such, the distribution and profile of migrants' countries-of-origin in Singapore and many parts of Asia also differs significantly from low TB burden host countries in Europe, Canada and USA, where migrants are predominantly from the Indian subcontinent and sub-Saharan Africa. While overall LTBI prevalence among Singapore residents was previously estimated at $12.7 \%,{ }^{9}$ detailed estimates are not available for migrants from key source countries who apply to work or live in Singapore.

The BCG vaccine, which is routinely administered in most countries with high TB prevalence and Singapore, confounds the interpretation of tuberculin skin test (TST) results, ${ }^{10}{ }^{11}$ therefore, screening of LTBI will be done through interferon-gamma release assay (IGRA) testing and not the TST.

In this study, we aim to estimate the prevalence of LTBI in migrants from high TB burden countries to Singapore, and evaluate the cost-effectiveness of an LTBI screening and treatment programme for these migrants, stratifying by their country-of-origin and other relevant demographic variables. The results from this study will be important for medium TB burden countries, and those that have large numbers of immigrants from these source countries.

\section{METHODS}

\section{Cross-sectional study}

Study design

To investigate the prevalence of LTBI in migrants originating from different countries, we performed a crosssectional study from May 2016 to January 2019 on workers from countries who contribute the majority of Singapore's migrant labour, since the main immigration inflows to Singapore are currently work related.

\section{Study sites and population}

Participants were recruited from 27 locations around Singapore. This included clinics providing health screening services for migrant workers, worker dormitories and recreation centres catering to migrant workers. Individuals from Bangladesh, China, India, Indonesia, Malaysia, Myanmar, Philippines and Vietnam were eligible if they were: (1) aged between 20 and 50 years; (2) had not previously worked in Singapore; (3) had stayed in Singapore for less than 1 year to be representative of the Singapore migrant population and to reduce the likelihood of including LTBI acquired in Singapore.

\section{Patient and public involvement}

Participants of the study were not involved in the design, or conduct, or reporting, or dissemination plans of our research.

\section{Study procedures}

All study team members were trained according to the protocol to ensure consistency and compliance with study procedures. Informed consent was obtained and documented prior to study commencement. We used interviewer-administered questionnaires to collect data on demographics, past and present living conditions, medical history and lifestyle behaviours; and collected blood samples.

\section{Sample processing and laboratory analysis}

Blood samples were sent to Tan Tock Seng Hospital on the day of collection to test for LTBI using QuantiFERON-TB Plus (QIAGEN) IGRA. Samples with indeterminate results were not retested. We considered all participants who were asymptomatic but tested positive using IGRA as having LTBI.

\section{Data analysis}

The multivariable modified Breslow-Cox proportional hazards model was used to identify independent risk factors for LTBI. The model was fitted using LTBI as the outcome with covariates significant at $\mathrm{p}<0.10$ used in multivariable analysis to account for potential suppressor variables when adjusting for confounding. Covariates identified from literature as known LTBI risk factors were also included.

\section{Approach to cost-effectiveness analysis}

We evaluated the cost-effectiveness for adopting LTBI screen-and-treat for groups of migrants applying for longterm stay on entry to Singapore, using interferon-gamma IGRA. We used the TreeAge software (version 2019, TreeAge Corp), to model a hypothetical cohort of 10000 new migrants aged 25 years to Singapore (figure 1). We compared a one-step IGRA screen-and-treat strategy to the current status quo of no screening over a 50 year time horizon, from a government payer perspective. Several current LTBI treatment regimens were considered and the most cost-effective treatment were used for subsequent analysis. We chose a 50-year time horizon to simulate the approximate life expectancy (LE) of migrants of 75 years old assuming all migrants enter at aged 25 years and stay for life thereafter. Both cost and outcomes were discounted at an annual rate of $3 \%$. Outcomes included the number of active TB case averted, discounted qualityadjusted life-years (QALYs), reduced medical costs and ICERs.

Our base case assumed the starting age of 25 years old. The branches of the decision tree captured the prevalence of LTBI, the probability of testing negative (including false negatives) or positive by IGRA (including false positives) and thus initiating LTBI preventive treatment, and on treatment the probability of treatmentinduced hepatoxicity. At the ends of each branch in the decision tree, individuals were passed through a Markov model for a yearly cycle of active TB progression rates from latent TB. This simulated, with age-specific rates, 


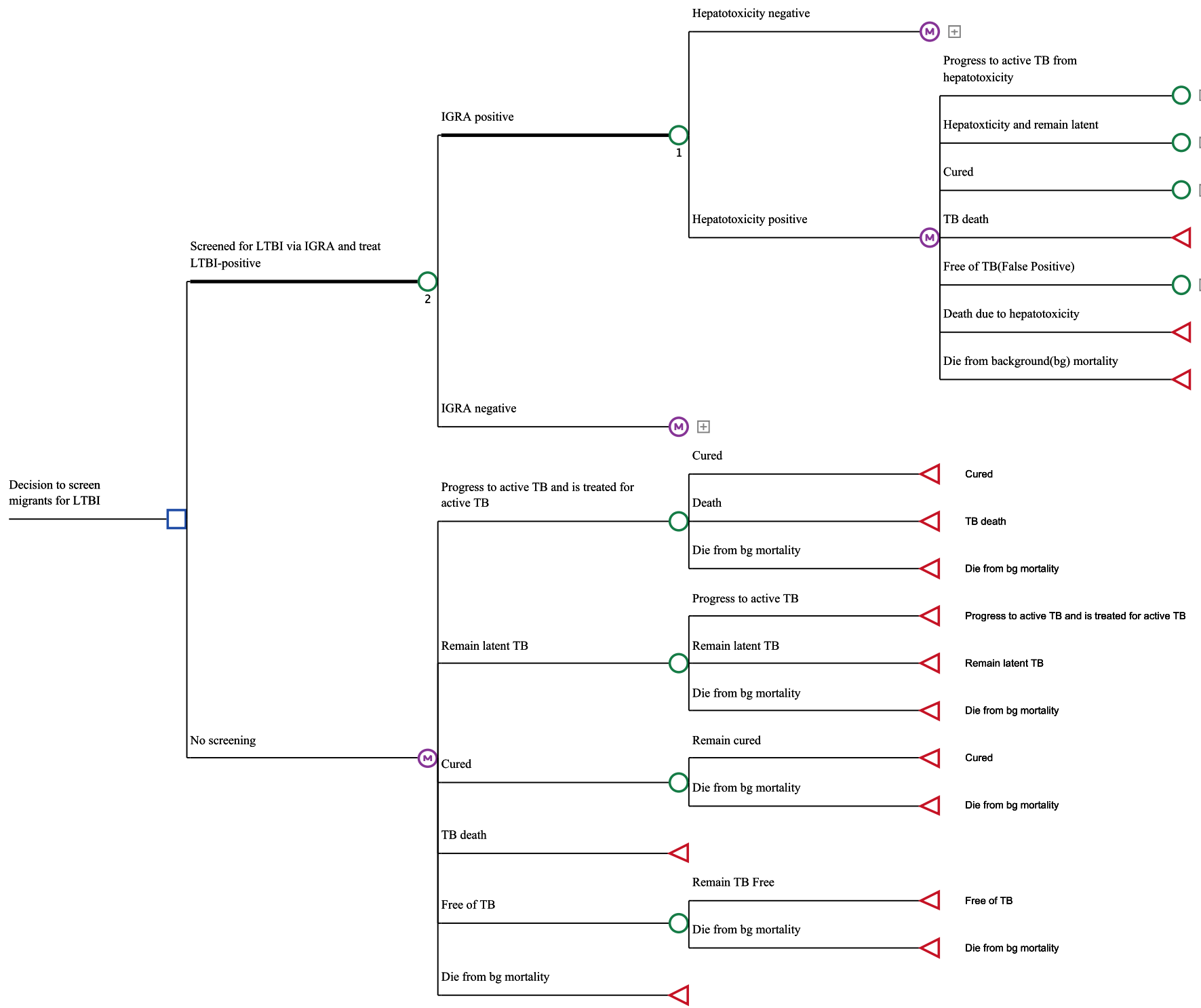

Figure 1 Decision tree model for the cost-effectiveness analysis. Markov downstream states not shown follows a similar profile from the other Markov nodes. IGRA, interferon-gamma release assay; LTBI, latent tuberculosis infection.

long-term outcomes related to the natural history of LTBI, including (1) LTBI progression to active TB, (2) mortality from active TB and (3) background mortality (see online supplemental material 1).

\section{Input parameters}

Input parameters on the probabilities of outcomes of screening and management of latent and active TB were obtained from published literature in PubMed/ MEDLINE, Cochrane and reports and guidelines published by international health authorities (eg, WHO), where available, and inputs provided by Singapore's Tuberculosis Control Unit (table 1).

Costs of screening and management of latent and active TB included costs of the LTBI screening test, evaluation to exclude active TB, LTBI treatment, active TB treatment, as well as the downstream cost of TB contact investigation into active TB cases. All treatment and clinical evaluation costs included consultation, medication and relevant laboratory and radiological investigations. Both costs and outcomes were discounted at an annual rate of $3 \%$. To determine whether the screening was costeffective, we compared the cost per QALY gained to a locally appropriate willingness to pay (WTP) threshold of S\$\$80000 per QALY (approximately the per capita gross domestic product in Singapore). Interventions below this threshold were considered as a possible efficient allocation of healthcare resources. Using this WTP value, we also estimated the length of stay (LOS) threshold for screen-and-treat to be cost-effective.

We also performed multiple one-way sensitivity analysis to evaluate the uncertainty around model parameters, based on reported CIs as plausible ranges for the parameters in published literature. Where Cls were unavailable, the sensitivity analysis covers a range of 0.5 times to 2 times of the base-case estimates. 
Table 1 Input parameters for the cost-effectiveness analysis

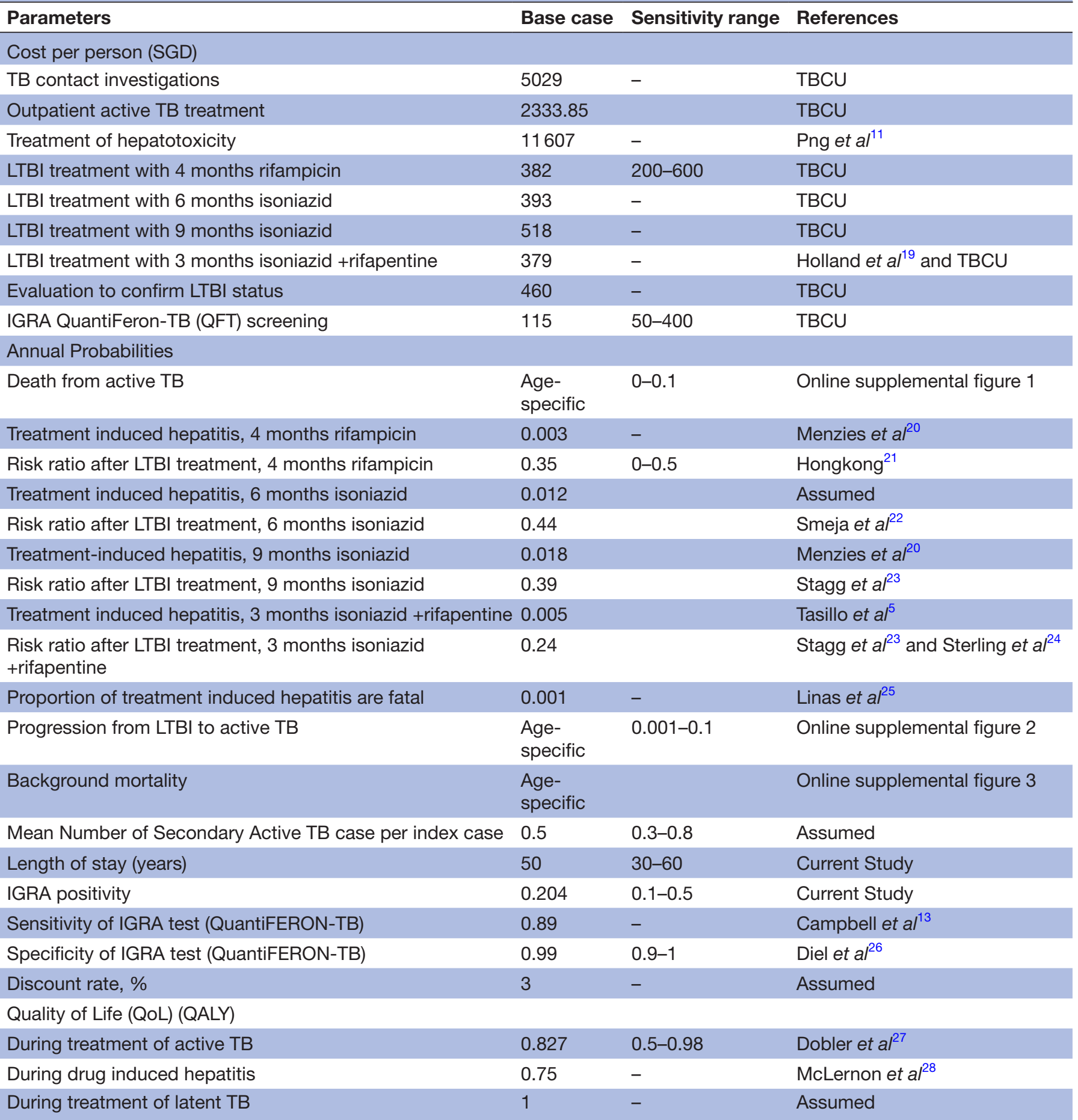

IGRA, interferon-gamma release assay; QALY, quality-adjusted life years; SGD, Singapore dollars; TB, tuberculosis; TBCU, tuberculosis control unit.

\section{RESULTS}

\section{Participant characteristics}

We recruited 3618 migrants, of which 34 were excluded from further analysis-19 due to indeterminate IGRA results, 2 born outside the countries of interest (1 each from Singapore and Brunei) and 13 who reported symptoms that may be indicative of active TB. Of the 3584 observations analysed, 727 (20.4\%) migrants had a positive IGRA result (table 2).

\section{Risk factors associated with LTBI}

On multivariable regression (table 2), migrants aged 30-39 years were significantly more likely to be LTBI positive (adjusted HR (aHR) 1.33 95\% CI 1.09 to 1.62; $\mathrm{p}=0.004$ ) 


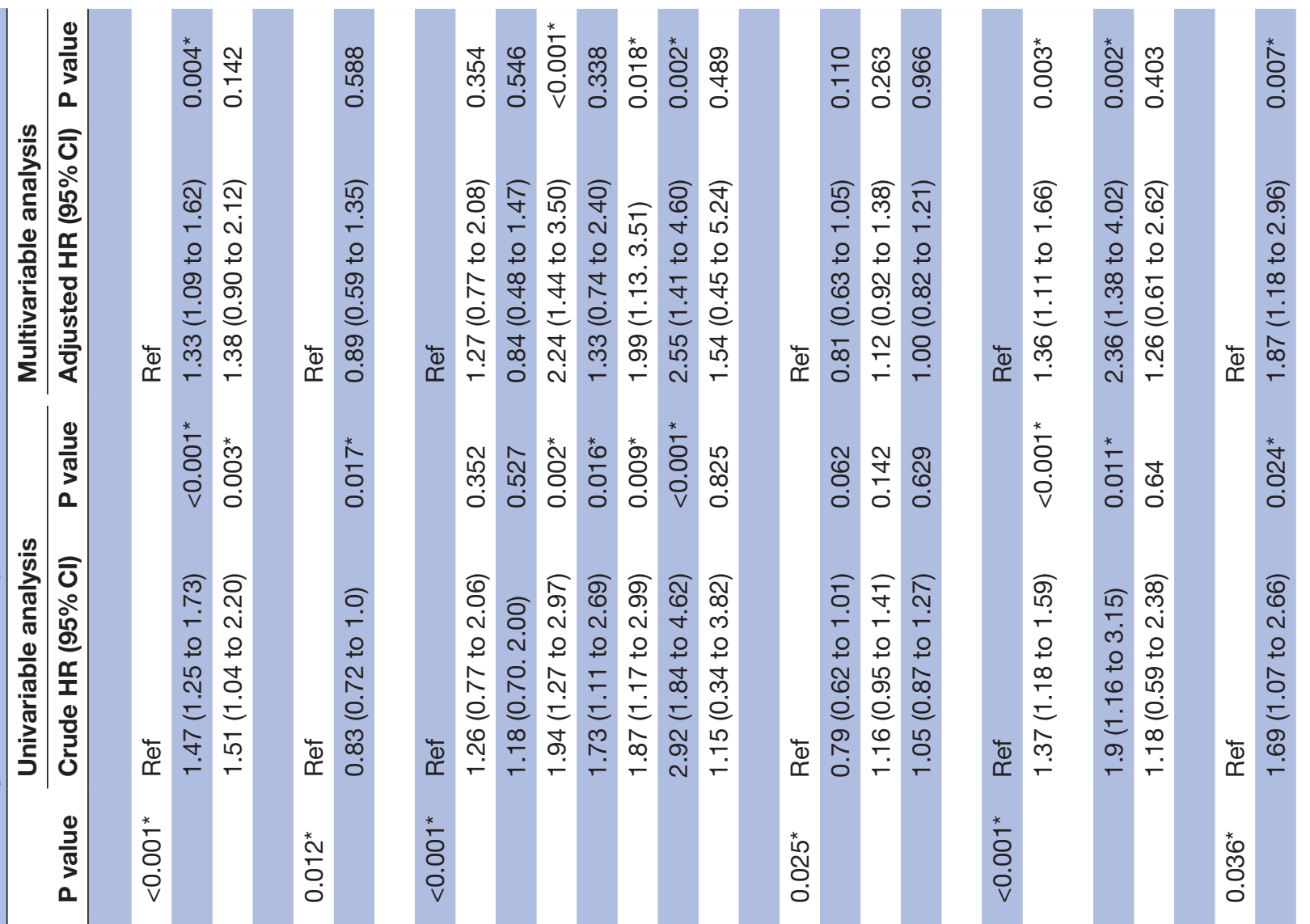

$\sum_{\pi}^{0}$

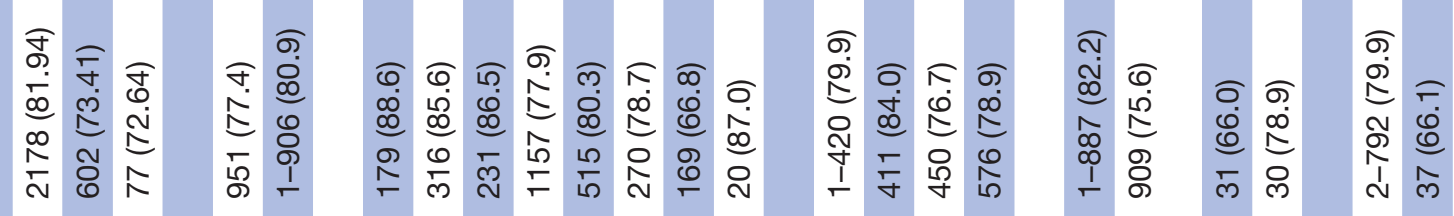

ช ช เฺ

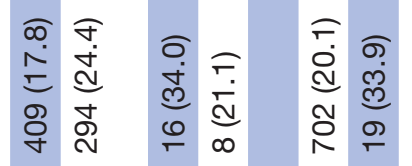

z

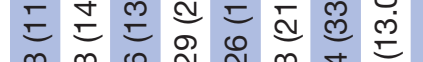

¿ิ่

邑

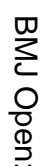

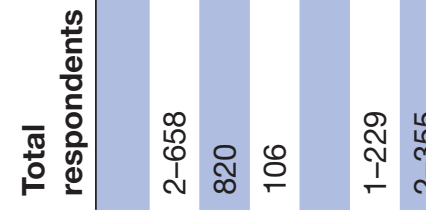

峞

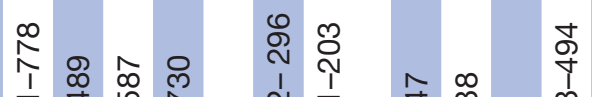

令总
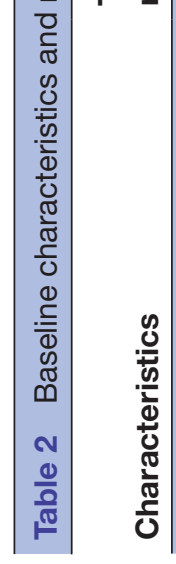

$\frac{5}{9}$

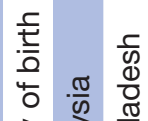

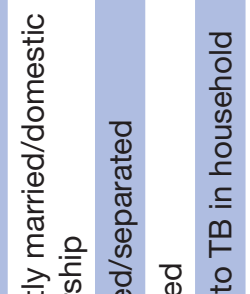

$\underset{\substack{0 \\ \frac{\pi}{0}}}{\stackrel{0}{0}}$ क क 
than those aged 20-29 years, and currently married (aHR $1.3695 \%$ CI 1.11 to $1.66 ; 0.003$ ) and divorced/separated (aHR 2.36 95\% CI 1.38 to 4.02; $\mathrm{p}=0.002$ ) individuals were more likely to be LTBI positive than single individuals. Self-reported past exposure to active TB in the household was significantly associated with LTBI (aHR 1.87, 95\% CI 1.18 to 2.96; $\mathrm{p}=0.007)$. Migrants from India, Myanmar and Philippines were significantly $(\mathrm{p}<0.05)$ more likely to be LTBI positive compared with Malaysians.

\section{Base-case analysis: cost-effectiveness of screening and treating migrants coming to Singapore}

With the current practice (no interventions), for a hypothetical cohort of 10000 new migrants coming to Singapore, the model predicted 234 cases of active TB disease, which cost $S \$ 1563135$ for treatment and public health interventions (table 3). The most cost-effective LTBI treatment regimens would be 3 months once weekly isoniazid (INH) and rifapentine combination regimen, which would prevent 156 active TB cases per 10000 migrants over a 50 -year time horizon, followed closely by 4 months rifampicin, then 6 months INH and 9 months INH. We further evaluated the most cost-effective regimen in the rest of our analysis.

Screen-and-treat could therefore be cost-effective for migrants with IGRA positivity $20 \%$ entering Singapore at age 25 years old who stayed for a period of 50 years, with threshold LOS for cost-effectiveness being 44 years and a ICER of S\$57116QALY. Country specific costeffectiveness analysis with different IGRA positivity is shown in online supplemental table 1.

Figure 2 uses age-specific values for IGRA positivity from the different countries (including Singapore-born residents from, ${ }^{9}$ but excluding Vietnam due to insufficient sample size for age-stratified analyses). Although Malaysia has an LTBI prevalence similar to Singapore, migrants aged 30-39 years old had high LTBI prevalence and consequently an LOS threshold below 40 years. Indonesia, India, Myanmar and Philippines had LOS thresholds less than the imposed LE cap (75 years) across all age groups, with older age groups generally having a higher IGRA positivity and lower LOS threshold.

Legend: age group (number of participants), LOS, LE. LE was capped at 75 years old, and the mean age of the range was used in all analysis. For screen-and-treat to be cost-effective in the lifetime of the migrants, the LOS threshold has to be lower than the threshold imposed by the LE cap (dotted line).

\section{Sensitivity analysis}

In multiple one-way sensitivity analysis (figure 3), ICER was most sensitive to the cohort's LOS in Singapore, yearly disease progression rate from LTBI to active TB disease followed by the cost of IGRA testing. Increases in mortality due to active TB, IGRA positivity and progression rates to active TB were associated with decreases in ICER values. Conversely, greater effectiveness of preventive treatment (ie, lower risk ratios) and lower utility associated with 
active TB treatment and lower cost of IGRA testing was associated with decrease in ICER values. Other parameters that influenced ICER values included cost of LTBI treatment regimen, mean number of secondary active TB cases and the specificity of the IGRA test.

We also modelled how results may be affected by multidrug resistant (MDR) LTBI, based on WHO's estimates on the country specific proportions of MDR-TB among active TB cases (online supplemental figure 4) ${ }^{12}$ Results differed substantially based on our assumptions on whether preventive treatment would be effective or ineffective in reducing the progression to MDR-TB in LTBI due to MDR strains (online supplemental figure 5). For instance, migrants from China had a relatively low IGRA positivity of $13.5 \%$, but a high proportion of MDR-TB $(7.1 \%)$. The LOS threshold decreases from 49 years when ignoring the effect of MDR-TB to 39 years when preventive treatment is assumed to be equally effective for MDR LTBI as for non-MDR LTBI, with a corresponding change in ICER values from $\mathbf{S} \$ 77396$ to $\mathbf{\$} \$ 48990$ per QALY. When more than $5 \%$ of TB is MDR, screen-and-treat is cost-effective even for IGRA positivity of $10 \%$ if preventive treatment is effective (online supplemental figure $6 \mathrm{~A})$, but cost-effective only if IGRA positivity is greater than $20 \%$ if ineffective for MDR TB (online supplemental figure $6 \mathrm{~B}$ ).

\section{DISCUSSION}

In this study, we determined a much higher IGRA positivity in migrants $(20.4 \%)$ as compared with Singapore residents $(12.7 \%) .{ }^{9}$ The wide variation of LTBI prevalence by country of birth, which likely reflected longitudinal TB incidence rates in those countries, had a substantial impact in determining if a screen-and-treat strategy would be cost-effective in different groups of migrants.

The significant associations of age, country of birth, past exposure to TB, marital status with increased IGRA positivity were not unexpected. ${ }^{2}{ }^{13}$ IGRA positivity increased with age, and while the oldest age group did not have the highest IGRA positivity in some countries (figure 2), this could be due to the small number observations in that age group. Past household exposures to TB is a well-known risk factor for LTBI, ${ }^{14}$ and may also be a proxy for factors facilitating TB acquisition like lower socioeconomic status and overcrowding ${ }^{15}$; the convergence of these factors may also be behind observed associations with marital status.

Universal screen-and-treat for migrants to Singapore with once weekly INH and Rifapentine combination regimen had an ICER of $\$ \$ 57116$ per QALY compared with the current practice of no interventions, and could reduce the number of active TB cases by at least $50 \%$ for base case parameters with migrants staying 50 years in Singapore when they enter at age 25 years old.

This is comparable to results from a study from the United States which estimated US $\$ 83000$ per QALY per lifetime of each migrant entering USA at 35 years old. ${ }^{5}$ Another study from Canada reported that IGRA 
Singapore

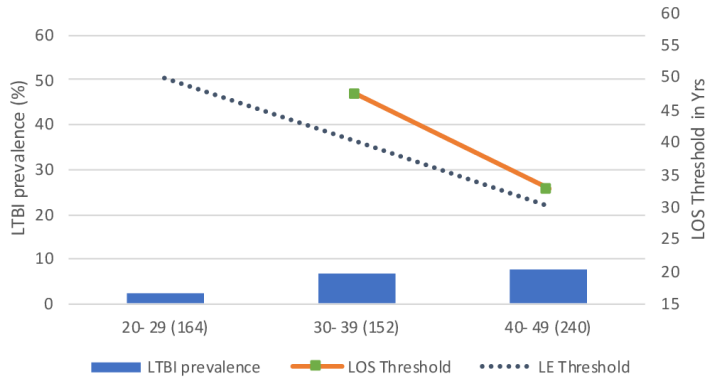

China

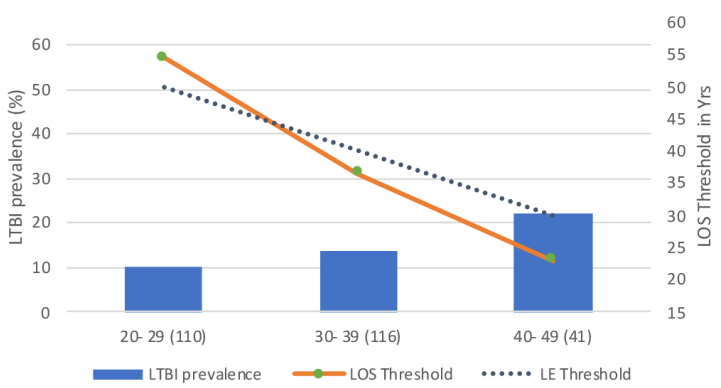

Bangladesh

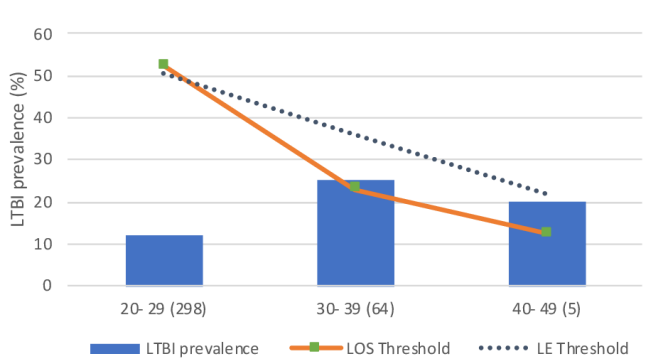

India

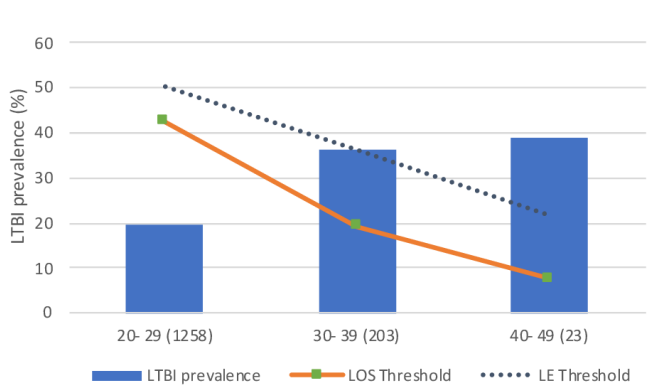

Malaysia

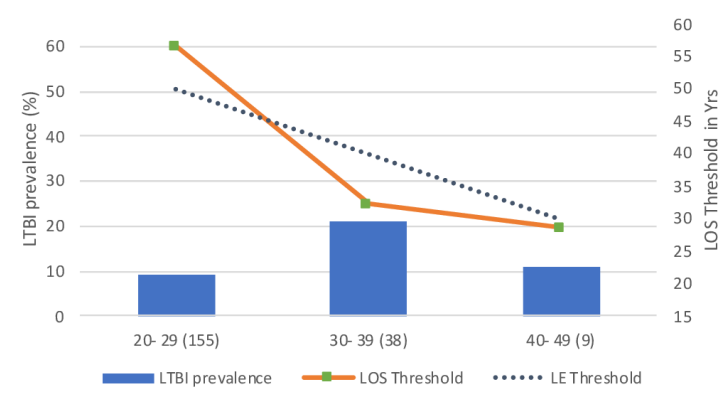

Myanmar

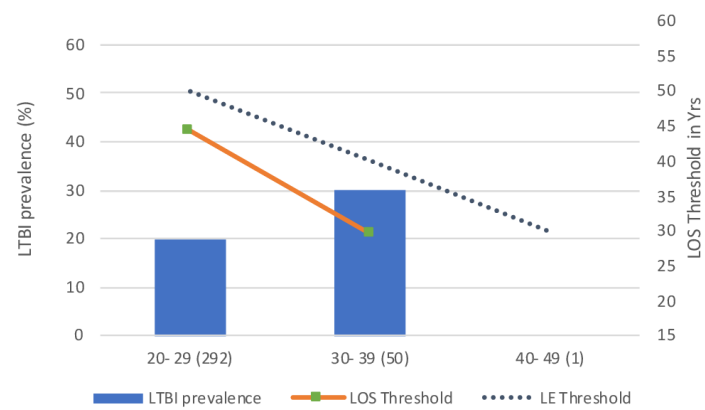

Indonesia

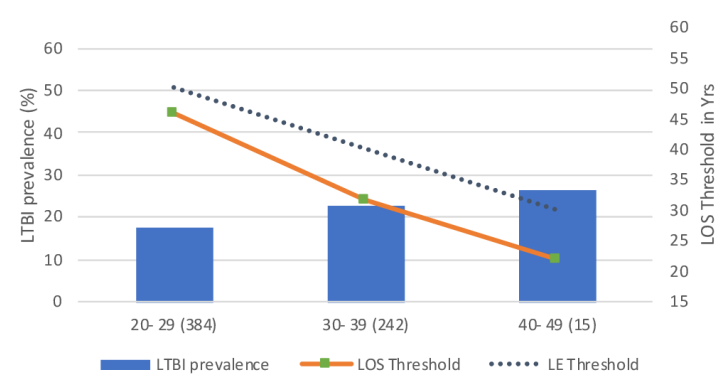

Philippines

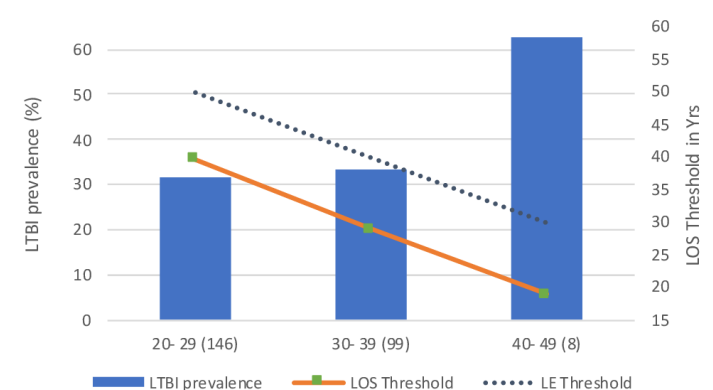

Figure 2 Country-specific length of stay thresholds for different LTBI prevalence in different age groups. LOS, length of stay; LTBI, latent tuberculosis infection.

screening coupled with rifampicin treatment for migrants from high incidence countries (LTBI prevalence about $20 \%$ ) gave a much lower ICER of $\$ 27200$ per QALY over a 25-year time horizon. ${ }^{4}$ Our assumption that mortality rates for active $\mathrm{TB}$ are low in younger individuals but increases with age accounts for some of these differences; had we assumed that $\sim 5 \%$ of TB cases will die regardless of age, then our ICER would be $\mathbf{\$} \$ 45231$ per QALY over a 25-year time horizon. As for the cost per TB case averted, we estimated an ICER of about $\mathbf{S} \$ 12421$ per active TB case averted over 50 years, and $\$ \$ 37288$ per active TB case for a 20-year time horizon, with the latter similar to the cost from a UK study for the same time horizon (20819 GBP, equivalent to $\$ \$ 36712$ per TB case averted).

However, given the variation in LTBI prevalence by country of origin and age, we also investigated the costeffectiveness of more targeted approaches. Screen-andtreat is cost-effective for migrants aged 25 years old from Indonesia, Myanmar, India and Philippines with a 50-year LOS in Singapore. However, the critical factor here was how long these migrants might stay. Notably, many migrants from the countries with higher LTBI prevalence 


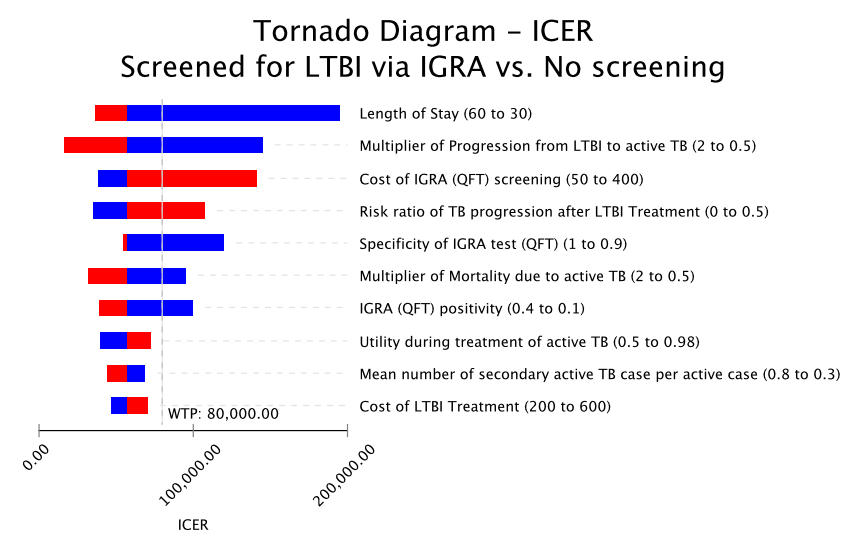

Figure 3 Tornado diagram for sensitivity analysis of selected parameters on ICER (S\$ per QALY). ICER, incremental cost-effectiveness ratio; IGRA, interferongamma release assay; LTBI, latent tuberculosis infection; QFT,QuantiFeron-TB; QALY, quality-adjusted life-year; WTP, willingness to pay.

do not stay for the periods needed to make the intervention cost-effective. For instance, a previous crosssectional study of male foreign migrant workers from India, Myanmar and Bangladesh living in a dormitory in Singapore found that $39 \%$ had stayed 2 or less years in Singapore, $17 \%$ had stayed 3-4 years, while $44 \%$ had been in Singapore for 5 or more years, ${ }^{16}$ which is substantially below the LOS thresholds for all nationalities and age groups. The wide variability in the time that migrants will reside in Singapore makes it challenging to base a screen-and-treat strategy on estimated LTBI prevalence alone, given the critical influence of LOS on whether the intervention is cost-effective. The projected LOS is in turn likely associated with industry and skill-set related factors for migrant workers.

Other than LOS, one-way sensitivity analysis found that the ICER was substantially affected by effectiveness of LTBI treatment, mortality due to TB, rates of disease progression among LTBI-infected individuals and the cost of LTBI screening via IGRA. Hence, in addition to identification of groups for screen-and-treat (eg, universal vs stratified by LTBI prevalence and projected LOS), reducing the costs associated with screening and management of LTBI could reduce the ICER and render the intervention more cost-effective.

Finally, when modelling how our results may be affected by the prevalence of MDR-TB, findings were critically influenced by assumptions about whether or not preventive treatment is effective in MDR LTBI. This remains unclear, but will hopefully be answered by results of the PHOENIx MDR TB trial, which looks at the efficacy and safety of 26 weeks of delamanid vs 26 weeks of INH for preventing confirmed or probable active TB during 96 weeks of follow-up among high-risk household contacts of adults with MDR-TB. ${ }^{17}$

Overall, our study suggests that a risk-stratified approach based on LTBI prevalence, age and projected LOS in Singapore as guided by the country of origin, occupational risk, and a combination of these factors would be more cost-effective than universal LTBI screenand-treat for new migrants. However, the average LOS from migrants to Singapore would render universal screen-and-treat strategies not to be cost-effective. Riskstratified approaches have been implemented elsewhere. For example, the National Institute for Health and Care Excellence in the UK recommends LTBI screening for new migrants aged 16-35 years from sub-Saharan Africa or from countries with a TB incidence of 150 cases per 100000 or higher. ${ }^{18}$ Another alternative to targeting all migrants on entry would be to screen-and-treat migrants from high TB incidence countries only when they apply for permanent residency or citizenship, as this would be accompanied with some certainty of a long LOS. These individuals may also be older, which would makes the intervention more cost-effective. However, this would miss the opportunity of preventing active TB cases arising in the earlier years of their stay.

Limitations in data and information availability required certain assumptions to facilitate the implementation of our model. For instance, sensitivity analyses found that the ICER was sensitive to the rates of disease progression to active TB. While internationally cited figures indicate progression rates of $5 \%-10 \%$ over the course of an individual's lifetime, with half occurring in the first 2years after infection, it is unknown when individuals were infected, and the rate of disease progression in migrants after entry into Singapore is also unknown. If some of these individuals with LTBI acquired TB infection just prior to entering Singapore, then the risks of disease progression would be higher than assumed and make screen-and-treat more cost-effective. Additionally, while certain assumptions such as maximising the proportion tested by IGRA who actually proceed to preventive treatment are within the control of policy instruments, others such as the prevalence of medical conditions contraindicating preventive treatment, and the probability that individuals could be infected with RIF-resistant strains and the effect of preventive treatment in such individuals remain unclear.

Despite the limitations, our model has identified some key factors that determine the cost-effectiveness of LTBI screening in migrants to Singapore. Any decision to implement universal LTBI screening for migrants must account for profiles of the cohort such as comorbidities, drug resistance but most importantly projected LOS. Additionally, policymakers should consider the impact on health budgets and the health system's capacity to support such an intervention.

In conclusion, we estimated LTBI prevalence for migrants to Singapore from key countries, and showed that the cost of preventing an active TB case or cost per QALY was high. Universal LTBI screening and treatment is not cost-effective at the average LOS for migrants. A risk-stratified approach by projected LOS, age and LTBI prevalence in country of origin would be a more cost-effective measure than universal screening and treatment of LTBI in all migrants at point of entry to Singapore. 


\section{Author affiliations}

${ }^{1}$ Infectious Disease Research and Training Office, National Centre for Infectious Diseases, Singapore

${ }^{2}$ Saw Swee Hock School of Public Health, National University of Singapore and National University Health System, Singapore

${ }^{3}$ Communicable Diseases Division, Ministry of Health Singapore, Singapore

${ }^{4}$ Tuberculosis Control Unit, Singapore TB Elimination Programme, Singapore

${ }^{5}$ Department of Laboratory Medicine, Tan Tock Seng Hospital, Singapore

Acknowledgements The study team would like to thank our collaborators from the various foreign worker dormitories, screening clinics and venues frequented by workers, for their help in recruiting and reaching out to our target population. We would also like to express our appreciation for all the migrant workers who took part in our study and shared valuable information with us.

Contributors VWL, HLW, PL, LWL, CEBC, RT-HO, VL and MC did the costeffectiveness analysis. YL and VWL did the prevalence and risk factor analysis. CBEC and VL provided access to the data for cost-effectiveness analysis. YRT, MXT, PY, TB and MC were in charge of the study design and data collection of the cross-sectional study. TB did the laboratory assays. All authors read and approved the final manuscript.

Funding This study was funded by the Communicable Diseases Public Health Research Grant: TR15NMR106 under the Ministry of Health, Singapore. Ministry of Health took part in the study design, provided data on tuberculosis and costs associated with tuberculosis treatments.

Competing interests None declared.

Patient consent for publication Not required.

Ethics approval The National University of Singapore Institutional Review Board approved this study (references 15-295 and S-18-390E).

Provenance and peer review Not commissioned; externally peer reviewed.

Data availability statement Data are available on reasonable request.

Supplemental material This content has been supplied by the author(s). It has not been vetted by BMJ Publishing Group Limited (BMJ) and may not have been peer-reviewed. Any opinions or recommendations discussed are solely those of the author(s) and are not endorsed by BMJ. BMJ disclaims all liability and responsibility arising from any reliance placed on the content. Where the content includes any translated material, BMJ does not warrant the accuracy and reliability of the translations (including but not limited to local regulations, clinical guidelines, terminology, drug names and drug dosages), and is not responsible for any error and/or omissions arising from translation and adaptation or otherwise

Open access This is an open access article distributed in accordance with the Creative Commons Attribution Non Commercial (CC BY-NC 4.0) license, which permits others to distribute, remix, adapt, build upon this work non-commercially, and license their derivative works on different terms, provided the original work is properly cited, appropriate credit is given, any changes made indicated, and the use is non-commercial. See: http://creativecommons.org/licenses/by-nc/4.0/.

\section{ORCID iDs}

Timothy Barkham http://orcid.org/0000-0003-0975-2244

Mark Chen http://orcid.org/0000-0001-9369-5830

\section{REFERENCES}

1 World Health Organization. Guidelines on the management of latent tuberculosis infection. Geneva: World Health Organization, 2015.

2 Pareek M, Watson JP, Ormerod LP, et al. Screening of immigrants in the UK for imported latent tuberculosis: a multicentre cohort study and cost-effectiveness analysis. Lancet Infect Dis 2011;11:435-44.

3 Pareek M, Bond M, Shorey J, et al. Community-Based evaluation of immigrant tuberculosis screening using interferon $\gamma$ release assays and tuberculin skin testing: observational study and economic analysis. Thorax 2013;68:230-9.

4 Campbell JR, Johnston JC, Sadatsafavi M, et al. Cost-Effectiveness of post-landing latent tuberculosis infection control strategies in new migrants to Canada. PLoS One 2017;12:e0186778.
5 Tasillo A, Salomon JA, Trikalinos TA, et al. Cost-Effectiveness of testing and treatment for latent tuberculosis infection in residents born outside the United States with and without medical comorbidities in a simulation model. JAMA Intern Med 2017;177:1755-64.

6 Chee CBE, Reves R, Zhang Y, et al. Latent tuberculosis infection: opportunities and challenges: latent tuberculosis infection review. Respirology 2018;23:893-900.

7 Ministry of Health Singapore. Communicable diseases surveillance in Singapore 2017. Singapore: Ministry of Health Singapore, 2018.

8 United Nations Population Division, Department of Economic and Social Affairs. International migrant stock: the 2017 revision, 2019. Available: https://www.un.org/en/development/desa/population/ migration/data/estimates2/estimates17.asp

9 Yap P, Tan KHX, Lim WY, et al. Prevalence of and risk factors associated with latent tuberculosis in Singapore: a cross-sectional survey. Int J Infect Dis 2018;72:55-62.

10 World Health Organization. Use of tuberculosis interferon-gamma release assays (IGRAs) in low- and middle- income countries: policy statement. Geneva: World Health Organization, 2011.

11 Png ME, Yoong J, Ong CWM, et al. A screening strategy for latent tuberculosis in healthcare workers: cost-effectiveness and budget impact of universal versus targeted screening. Infect Control Hosp Epidemiol 2019;40:341-9.

12 WHO. WHO | tuberculosis country profiles, 2019. Available: http:// www.who.int/tb/country/data/profiles/en/

13 Campbell JR, Krot J, Elwood K, et al. A systematic review on TST and IGRA tests used for diagnosis of LTBI in immigrants. Mol Diagn Ther 2015;19:9-24.

14 Chandrasekaran P, Mave V, Thiruvengadam K, et al. Tuberculin skin test and QuantiFERON-Gold in tube assay for diagnosis of latent TB infection among household contacts of pulmonary TB patients in high TB burden setting. PLoS One 2018;13:e0199360.

15 Lönnroth K, Jaramillo E, Williams BG, et al. Drivers of tuberculosis epidemics: the role of risk factors and social determinants. Soc Sci Med 2009;68:2240-6.

16 Lee W, Neo A, Tan S, et al. Health-seeking behaviour of male foreign migrant workers living in a dormitory in Singapore. BMC Health Serv Res 2014:14:300.

17 ClinicalTrials.gov. Protecting households on exposure to newly diagnosed index multidrug-resistant tuberculosis patients - full text view - ClinicalTrials.gov, 2019. Available: https://clinicaltrials.gov/ct2/ show/NCT03568383

18 Public Health England. Collaborative tuberculosis strategy for England: 2015 to 2020. London: Public Health England, 2015.

19 Holland DP, Sanders GD, Hamilton CD, et al. Costs and costeffectiveness of four treatment regimens for latent tuberculosis infection. Am J Respir Crit Care Med 2009;179:1055-60.

20 Menzies D, Adjobimey M, Ruslami R, et al. Four months of rifampin or nine months of isoniazid for latent tuberculosis in adults. $N$ Engl $J$ Med 2018;379:440-53.

21 A double-blind placebo-controlled clinical trial of three antituberculosis chemoprophylaxis regimens in patients with silicosis in Hong Kong. Hong Kong chest Service/Tuberculosis research centre, Madras/British medical Research Council. Am Rev Respir Dis 1992;145:36-41.

22 Smieja M, Marchetti C, Cook D, et al. Isoniazid for preventing tuberculosis in non-HIV infected persons. Cochrane Database of Systematic Reviews 1999;86.

23 Stagg HR, Zenner D, Harris RJ, et al. Treatment of latent tuberculosis infection: a network meta-analysis. Ann Intern Med 2014;161:419-28.

24 Sterling TR, Villarino ME, Borisov AS, et al. Three months of rifapentine and isoniazid for latent tuberculosis infection. $N$ Engl J Med 2011;365:2155-66.

25 Linas BP, Wong AY, Freedberg KA, et al. Priorities for screening and treatment of latent tuberculosis infection in the United States. Am J Respir Crit Care Med 2011;184:590-601.

26 Diel R, Loddenkemper R, Nienhaus A. Evidence-Based comparison of commercial interferon-gamma release assays for detecting active TB: a metaanalysis. Chest 2010;137:952-68.

27 Dobler CC, Martin A, Marks GB. Benefit of treatment of latent tuberculosis infection in individual patients. Eur Respir $J$ 2015;46:1397-406

28 McLernon DJ, Dillon J, Donnan PT. Health-state utilities in liver disease: a systematic review. Med Decis Making 2008;28:582-92. 